\title{
Dissecting Two-Dimensional Ultraviolet Spectra of Amyloid Fibrils into Beta-Strand and Turn Contributions
}

\author{
Justo J. Rodriguez* and Shaul Mukamel \\ Department of Chemistry, University of California, Irvine, California 92697, United States
}

\begin{abstract}
We present an analysis of the contributions of various secondary structure elements of the amyloid $\beta$-protein to the twodimensional far ultraviolet (2DFUV) signal of an amyloid fibril model. The contributions of the turns and the $\beta$-strands are affected by the geometry of the backbone peptide amide $\pi \rightarrow \pi^{*}$ transition dipoles, the backbone interamide coupling in the excited state, and the exciton delocalization. These contributions are clearly distinguishable in the $x y x y-x y y x$ pulse polarization configuration. The differences are attributed to the smaller splitting of the exciton energies and the larger fluctuations of the geometry of the peptide amide $\pi \rightarrow \pi^{*}$ transition dipoles at the

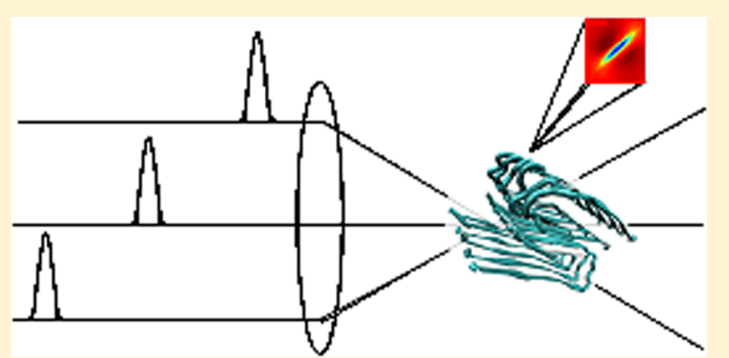
turns, while making the 2DFUV signal sensitive to the secondary structure. This signal may be used to determine the proportion of turns and $\beta$-strands.
\end{abstract}

\section{INTRODUCTION}

Absorption and circular dichroism spectra in the vacuum ultraviolet are commonly used to characterize the secondary structure of proteins. ${ }^{1}$ Circular dichroism is known to be highly sensitive to the spatial organization of backbone amide $n \rightarrow \pi^{*}$ and $\pi \rightarrow \pi^{*}$ transition dipoles and aromatic side chain transitions. ${ }^{2,3}$ Two-dimensional (2D) optical spectroscopy carries additional information. The extension of these techniques into the near-ultraviolet is now possible. ${ }^{4-9}$ Multidimensional spectroscopy in the middle and far ultraviolet could probe the backbone amide and aromatic side chains with high resolution, and facilitate their characterization. ${ }^{10}$

In this article, the 2DFUV signal is shown to reveal correlations of different secondary structure elements of the constituent proteins of amyloid fibrils. Specific combinations of pulse polarizations can be used to dissect the highly congested spectra of excitons. The $x y x y-x y y x$ polarization configuration has been used in two-dimensional infrared (2DIR) spectroscopy to eliminate diagonal peaks and increase the resolution of cross peaks. ${ }^{11}$ Polarization configurations have been used to measure the angles between chromophores in the CP29 light harvesting complex. ${ }^{12}$ Genetic algorithms have also been developed to construct optimal pulse polarizations and analyze selected features of simulated two-dimensional spectra of porphyrin dimers, ${ }^{13}$ and the Fenna-Matthews-Olson (FMO) photosynthetic complex. $^{14}$

These methods have been developed to dissect the contributions of different parts of the system to the two-dimensional spectrum. ${ }^{15}$ In the first method, a spectrum calculated by neglecting the couplings between the parts is compared with the coupled system. A second method, sensitivity analysis, ${ }^{16}$ shifts the energy of the elements of the Hamiltonian associated with a given chromophore or the coupling between them by a small amount and calculates the difference caused by this perturbation. A third dissection methodology is based on the projection, in real space, of the last laser pulse-matter interaction. ${ }^{15}$ In this dissection, the sum of the contributions from the various parts is equal to the total spectrum. These techniques are used in this article to identify the contributions of the turns and $\beta$-strands to the total $2 \mathrm{D}$ spectrum of the amyloid fibril model proposed by Tycko and co-workers. ${ }^{17}$

We have used the EHEF (exciton Hamiltonian with electrostatic fluctuations) algorithm ${ }^{10}$ for the simulation of the 2DUV spectrum. The simulation protocol is first described in the methodology. In section 3, we present the distribution of electronic transitions and geometry of the proteins. In section 4 , we analyze the extent of exciton delocalization across the protein and its correlation with the angle between neighboring backbone amide $\pi \rightarrow \pi^{*}$ dipoles. The contributions of turns and $\beta$-strands of the 2DFUV signal are analyzed in section 5 . Even though chiral signals have been shown to be very sensitive to the secondary structure of the protein, ${ }^{2,10}$ they are much weaker. In this article, we focus on the nonchiral $2 \mathrm{D}$ spectra.

\section{METHODOLOGY AND AMYLOID FIBRIL MODEL}

We use the molecular model of 32 -residue $\beta$-amyloid $\left(\mathrm{A} \beta_{9-40}\right)$ constructed by Tycko and co-workers. In this model based on NMR spectra, the conformation of the $\mathrm{A} \beta_{9-40}$ protein is an asymmetric " $\mathrm{U}$ " comprised of N-terminal and C-terminal $\beta$-strands, and a turn that links the strands. ${ }^{17}$ The proteins have three aromatic side-chains: Tyr10, Phe19, and Phe20, all on the $\mathrm{N}$-terminal strand. We used in our simulation an aggregate model composed of 12 native $\mathrm{A} \beta_{9-40}$ proteins (see Figure 1 ). The effective fluctuating QM Hamiltonian, transition dipoles, and coordinates of the chromophores were constructed using

Received: April 24, 2012

Revised: June 20, 2012

Published: June 27, 2012 


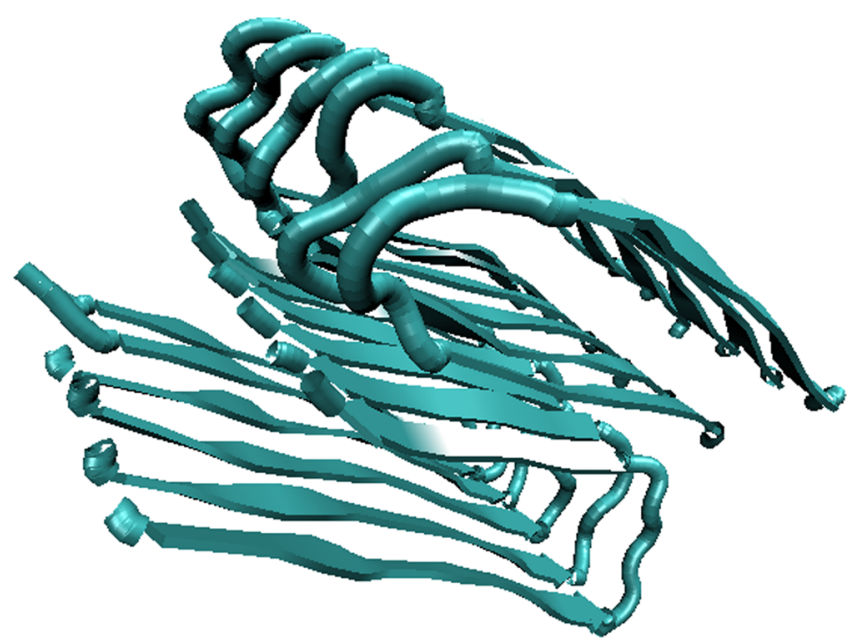

Figure 1. Amyloid fibril model of twelve 32-residue $\beta$-amyloids $\left(\mathrm{A} \beta_{9-40}\right)$. The structure is described in the methodology and in ref 17.

the matrix method in the DichroCalc core. ${ }^{18}$ Details of the molecular dynamics simulation are given in ref 6.300 snapshots at $310 \mathrm{~K}$ with 1 ps interval were harvested for the calculation of spectra.

Our simulation includes the backbone amide $n \rightarrow \pi^{*}$ $(220 \mathrm{~nm})$ and $\pi \rightarrow \pi^{*}(190 \mathrm{~nm})$ electronic transitions. Each aromatic side chain has four valence electronic excitations in the $>250 \mathrm{~nm}$ region, ${ }^{1} \mathrm{~L}_{\mathrm{b}},{ }^{1} \mathrm{~L}_{\mathrm{a}},{ }^{1} \mathrm{~B}_{\mathrm{b}}$, and ${ }^{1} \mathrm{~B}_{\mathrm{b}}$ in Platt's notation. ${ }^{19}$ The photon-echo signal was simulated using the nonlinear exciton equations. ${ }^{15}$ As implemented in the Spectron code, we have assumed Lorentzian linewidths with the same dephasing rate of $250 \mathrm{~cm}^{-1}$ for all transitions. The $2 \mathrm{D}$ spectra were obtained using four short Gaussian pulses with a frequency of $52000 \mathrm{~cm}^{-1}$, and a full width at half-maximum (fwhm) of $3754 \mathrm{~cm}^{-1}$ was used. The time delay between the second and third pulses is zero, and consequently, no energy transport is observed in the signals.

\section{ELECTRONIC COUPLINGS AND THE ANGLE BETWEEN $\pi \rightarrow \pi^{*}$ TRANSITION DIPOLES VS THE PEPTIDE BOND INDEX}

2DFUV spectra of proteins probe the couplings between backbone peptide amide $\pi \rightarrow \pi^{*}$ and $n \rightarrow \pi^{*}$ and the aromatic side chain transitions. Table 1 compares the magnitude of the transition dipoles and excited state energies for all transitions included in the simulations. A number of factors suggest that the $\pi \rightarrow \pi^{*}$ transitions dominate the correlations observed in the signal: (a) The $\pi \rightarrow \pi^{*}$ transition dipoles are much larger than the $n \rightarrow \pi^{*}$ dipoles; (b) the aromatic residues are rare; (c) the power spectrum of the pulses overlaps strongly with the $\pi \rightarrow \pi^{*}$ transitions; (d) neighboring $\pi \rightarrow \pi^{*}$ transition dipoles are strongly coupled (with off-diagonal elements $\sim 10^{3} \mathrm{~cm}^{-1}$ for the $\beta$-strand). Thus, we focus our analysis on the $\pi \rightarrow \pi^{*}$ transitions. The $x y x y-x y y x$ polarization configuration amplifies the features associated with strongly coupled transitions.

The interaction of the chromophores with local electrostatic fields shifts the energy of the exciton states, and these local fields vary considerably with geometry fluctuations. ${ }^{20}$ Figure $2 \mathrm{a}$ shows the distribution of the excited state electronic couplings between neighboring amide $\pi \rightarrow \pi^{*}$ transitions and its variation with the peptide bond $N$. Clearly, these couplings fluctuate much more strongly at the turns than at the $\beta$-strands. The couplings at the strands and the exciton splitting are generally
Table 1. Magnitude of the Transition Dipoles, Excited State Energies, and Number of Transitions in the Amyloid Fibril Model $^{a}$

$\begin{array}{lccc}\text { transition } & \begin{array}{c}\text { average excited } \\ \text { magnitude of } \\ \text { the transition } \\ \text { dipoles (D) }\end{array} & \begin{array}{c}\text { cransition } \\ \text { frequency of the } \\ \text { chromophore } \\ \left(10^{3} \mathrm{~cm}^{-1}\right)\end{array} & \begin{array}{c}\text { number of } \\ \text { transitions } \\ \text { in the model }\end{array} \\ \text { backbone amide } \pi \rightarrow \pi^{*} & 1.78 & 52 & 372 \\ \text { backbone amide } n \rightarrow \pi^{*} & 0.1 & 44 & 372 \\ \text { aromatic tyrosine }{ }^{1} \mathrm{~L}_{\mathrm{b}} & 0.1 & 37 & 12 \\ \text { aromatic tyrosine }{ }^{1} \mathrm{~L}_{\mathrm{a}} & 0.38 & 48 & 12 \\ \text { aromatic tyrosine }{ }^{1} \mathrm{~B}_{\mathrm{b}} & 6.7 & 52 & 12 \\ \text { aromatic tyrosine }{ }^{1} \mathrm{~B}_{\mathrm{a}} & 5.91 & 52 & 12 \\ \text { aromatic phenylalanine }{ }^{1} \mathrm{~L}_{\mathrm{b}} & 6.82 & 39 & 24 \\ \text { aromatic phenylalanine }{ }^{1} \mathrm{~L}_{\mathrm{a}} & 0 & & 24 \\ \text { aromatic phenylalanine }{ }^{1} \mathrm{~B}_{\mathrm{b}} & 0 & 48 & 24 \\ \text { aromatic phenylalanine }{ }^{1} \mathrm{~B}_{\mathrm{a}} & 6.82 & & \end{array}$

${ }^{a}$ See ref 10 for further details of the simulation and ref 17 for details of the model.
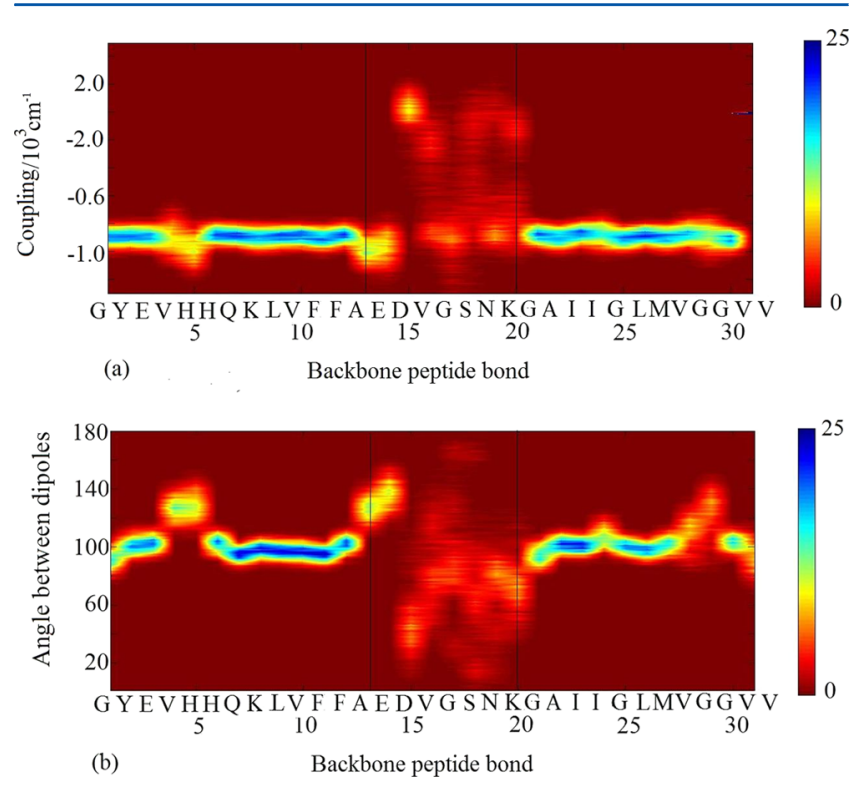

Figure 2. Distribution of excited state electronic couplings and angles between the amide $\pi \rightarrow \pi^{*}$ transitions of $\mathrm{A} \beta_{9-40}$ proteins averaged over $300 \mathrm{MD}$ snapshots in a $0.3 \mathrm{~ns}$ trajectory. The data are averaged over all $\mathrm{A} \beta_{9-40}$ proteins in the amyloid fibril model. $N$ represents the peptide bond as it appears in the amino acid sequence (shown at the bottom of the contour map). In part a, the occurrence (color scale) of the excited state electronic coupling between the amide $\pi \rightarrow \pi^{*}$ transitions ( $y$ axis) at peptide bonds $N$ and $N+1$ is shown at peptide bond $N$ (the couplings are grouped in $10 \mathrm{~cm}^{-1}$ segments). In part b, the occurrence (color scale) of angles ( $y$ axis) between neighboring $\pi \rightarrow \pi^{*}$ transition dipoles of peptide bonds $N$ and $N+1$ is shown at peptide bond $N$ (the angles are approximated as integers to calculate the occurrence on the color scale). The two vertical lines at residue 13 and 20 separate the regions associated with the $\beta$-strands and with the turns based on the angle between neighboring peptide transition dipoles.

stronger. The coupling between second neighboring chromophores is about $100 \mathrm{~cm}^{-1}$, and its contribution is much lower.

Figure $2 \mathrm{~b}$ shows the distribution of angles between $\pi \rightarrow \pi^{*}$ transition dipoles of neighboring peptide bonds as a function of the peptide bond index. These angles are centered around $90^{\circ}$ at the turns and strongly fluctuate due to the nuclear motion. 
At the $\beta$-strands, in contrast, the angles are almost constant, reflecting the more rigid geometry. In section 5 , we shall relate the distribution of backbone interamide couplings and transition dipole geometry with the contribution of various secondary structure elements to the $2 \mathrm{DFUV}$ signal.

\section{EXCITON DELOCALIZATION ALONG THE PROTEIN: THE ROLE OF THE AROMATIC SIDE CHAINS}

The participation ratio provides a convenient measure of an exciton size. ${ }^{21}$ Its inverse gives the number of chromophores on which the exciton is delocalized. For our model, this parameter is 3.4. We have calculated the following parameter which is a measure of the number of chromophores on which the excitons are delocalized within the local environment around a selected chromophore $j$

$$
M_{j}=\sum_{\mathrm{e}} \frac{\left|c_{j}^{\mathrm{e}}\right|^{2}}{\sum_{i}\left|c_{i}^{e}\right|^{4}}
$$

$c_{j}^{\mathrm{e}}$ is the coefficient of chromophore $j$ in the exciton state e. This can be used to track the extent of delocalization of the excitons along the proteins.

Figure 3 depicts $M_{j}$ at the $\pi \rightarrow \pi^{*}$ transitions. The excitons are more delocalized at the $\beta$-strands than at the turns. This can

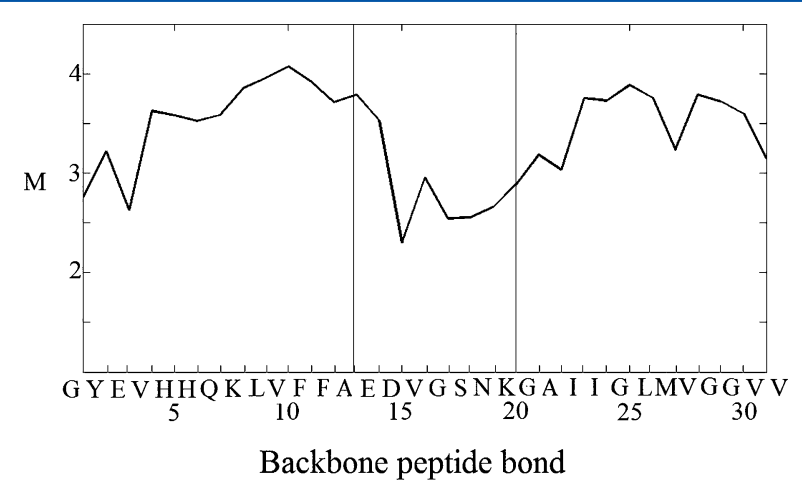

Figure 3. Measure of the number of chromophores on which the excitons are delocalized (eq 4.1) at the local environment of a $\pi \rightarrow \pi^{*}$ transition versus the peptide bond $N$.

be rationalized by two factors. First, a stronger coupling between almost antiparallel dipoles at the $\beta$-strands. Second, maximum delocalization is achieved close to the position of the two phenylalanines $(\mathrm{F})$.

In Figure 4, we depict a scatter plot of the average angle between neighboring amide $\pi \rightarrow \pi^{*}$ transitions vs the number of chromophores on which the excitons are delocalized (eq 4.1). It clearly separates into two spectral regions that can be

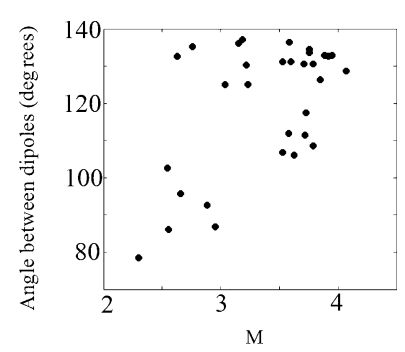

Figure 4. Scatter plot of average angle between neighboring transition dipoles and delocalization (eq 4.1). It shows two regions associated with the turns (angles $80-90^{\circ}$ ) and the $\beta$-strands (from about 110 to $140^{\circ}$ ). associated with the turns (from 80 to $90^{\circ}$ ) and the $\beta$-strands (from 110 to $140^{\circ}$ ). The lower delocalization at the turns reduces the overlap between excitons and the magnitude of their contribution to the 2DFUV signal.

In the following section, we proceed to analyze the $2 \mathrm{DFUV}$ signal based on these exciton properties.

\section{ANALYSIS OF THE 2DFUV SIGNAL}

The 2DFUV $x x x x$ spectrum shown in Figure 5a has a very narrow and intense blue peak along the diagonal, which is dominated by single exciton contributions and resembles the absorption spectrum. ${ }^{10}$ The red cross-peaks are much weaker and they are symmetric with respect to the diagonal. The $x y x y-$ $x y y x$ signal (Figure $5 b$ ) is 2 orders of magnitude weaker. The $x x x x$ signal is dominated along the diagonal, $\Omega_{3}=-\Omega_{1}$, by single exciton contributions, while $x y x y-x y y x$ amplifies correlations between excitons with parallel transition dipoles. The $x y x y-x y y x$ signal is much more sensitive to the geometry than $x x x x$. It shows a diagonal blue peak that is considerably broader. Single exciton contributions are eliminated by this pulse sequence. $^{11}$

\section{ANALYSIS OF THE DISSECTION MAPS}

We next discuss the contributions of the turns and $\beta$-strands of the $\mathrm{A} \beta_{9-40}$ proteins to the 2DFUV signals. The Frenkel exciton Hamiltonian for the complete aggregate is first divided into the turns and the $\beta$-strands. The contributions to the $x y x y-x y y x$ 2DFUV signal are shown in Figure $6 \mathrm{~b}$ for the turns and $6 \mathrm{c}$ for the strands. The spectrum for the separated $\beta$-strands is very similar to the complete spectrum with coupling, but the central peak is clearly split into two. This may be attributed to the strong coupling between transitions at the $\beta$-strands, leading to the splitting of the electronic transitions in blue. The red peaks are then generated by the couplings between split transitions of low and high energy at the $\beta$-strands.

The sum of the contributions of the turns and the $\beta$-strands obtained by neglecting their coupling is shown in Figure 6 a. This is very different from the spectrum for the complete amyloid fibril (Figures $5 \mathrm{~b}$ and $6 \mathrm{~d}$ ). This suggests a weakness in this dissection methodology. When the Hamiltonian of the whole aggregate is divided into two, some off-diagonal elements of the Hamiltonian are eliminated. Since the excitons at the turns are strongly coupled with the strands, the $2 \mathrm{D}$ spectrum of these separated fragments (Figure $6 \mathrm{~b}$ and $\mathrm{c}$ ) is not realistic. A different method based on the projection on real space of the last laser pulse-matter interaction in the four-wavemixing experiment has been used previously. ${ }^{15}$ In this dissection, the sum of the contributions is equal to the total signal. This gives Figure $6 \mathrm{e}$ and $\mathrm{f}$ for the $\beta$-strands and the turns, respectively. Figure $6 \mathrm{e}$ can be interpreted in a similar way as Figure $6 \mathrm{~b}$. However, Figure $6 \mathrm{f}$ is very different from Figure $6 c$. The spectrum for the turns, Figure $6 c$, is strongest at $52000 \mathrm{~cm}^{-1}$. No splitting is observed due to the lower coupling between neighboring peptide $\pi \rightarrow \pi^{*}$ transitions in these regions.

The sensitivity analysis ${ }^{20}$ contour maps were constructed by increasing the energy of the diagonal elements of the Hamiltonian that are associated with the turns, Figure $6 \mathrm{~h}$, or $\beta$-strands, Figure $6 \mathrm{i}$, by $0.1 \%$ and subsequently calculating the shift of the signal with this perturbation. The sensitivity map for the turns (Figure 6i) is strongest at 54000 and $55000 \mathrm{~cm}^{-1}$, while the largest sensitivity for the $\beta$-strands is observed at 


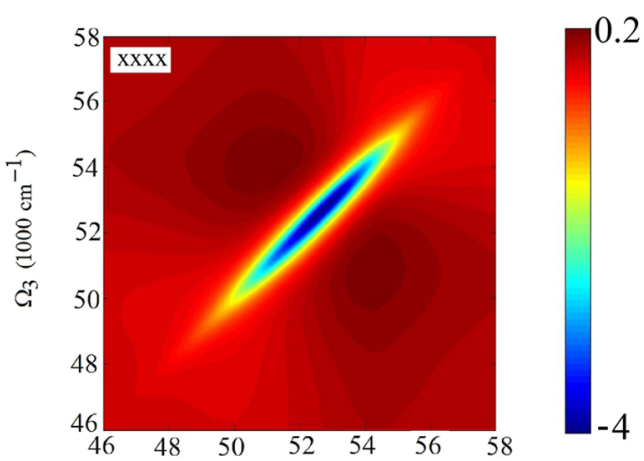

(a) $-\Omega_{1}\left(1000 \mathrm{~cm}^{-1}\right)$

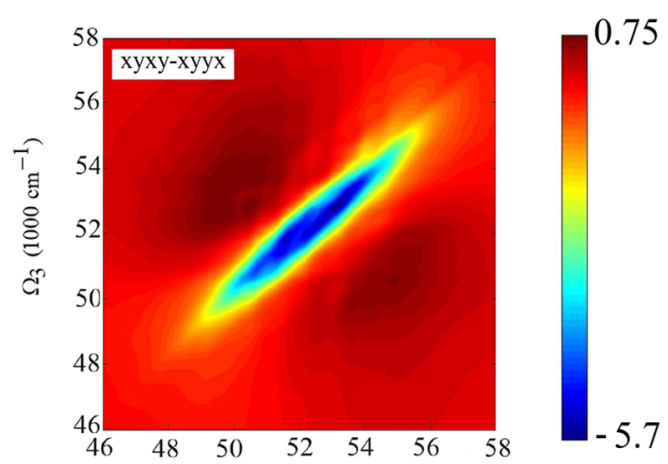

(b) $-\Omega_{1}\left(1000 \mathrm{~cm}^{-1}\right)$

Figure 5. 2DFUV contour maps for the amyloid fibril model: (a) $x x x x$ and (b) $x y x y-x y y x$. Part b is 2 orders of magnitude weaker.

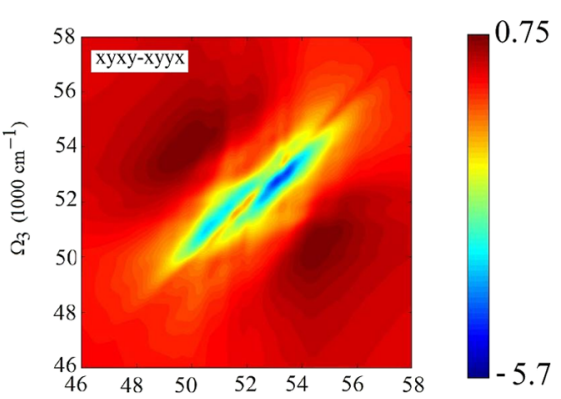

(a) $\quad-\Omega_{1}\left(1000 \mathrm{~cm}^{-1}\right)$

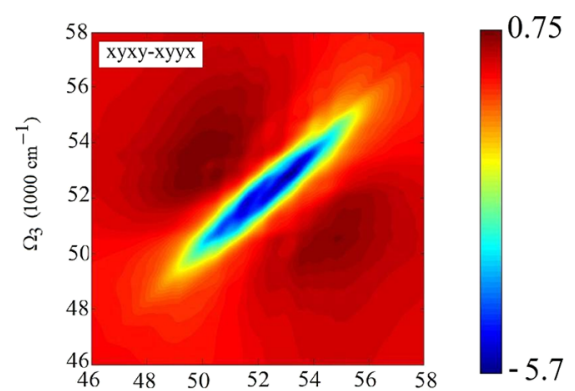

(d) $\quad-\Omega_{1}\left(1000 \mathrm{~cm}^{-1}\right)$

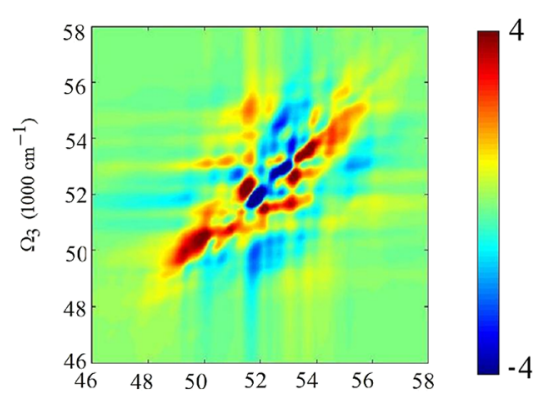

(g) $-\Omega_{1}\left(1000 \mathrm{~cm}^{-1}\right)$

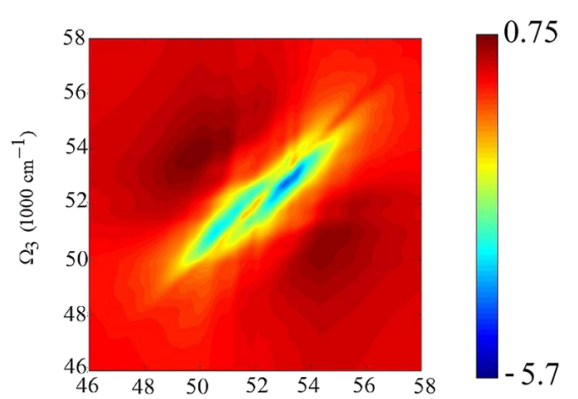

(b) $\quad-\Omega_{1}\left(1000 \mathrm{~cm}^{-1}\right)$

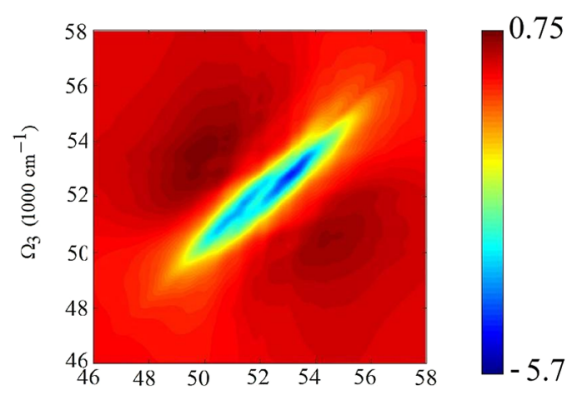

(e) $\quad-\Omega_{1}\left(1000 \mathrm{~cm}^{-1}\right)$

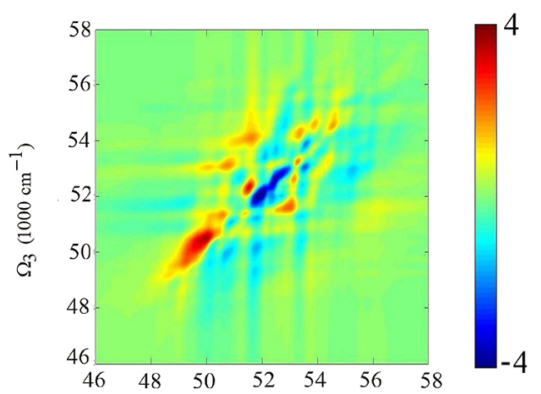

(h) $\quad \Omega_{1}\left(1000 \mathrm{~cm}^{-1}\right)$

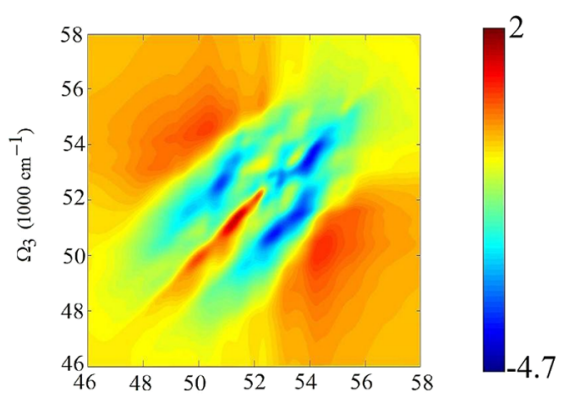

(c) $\quad-\Omega_{1}\left(1000 \mathrm{~cm}^{-1}\right)$

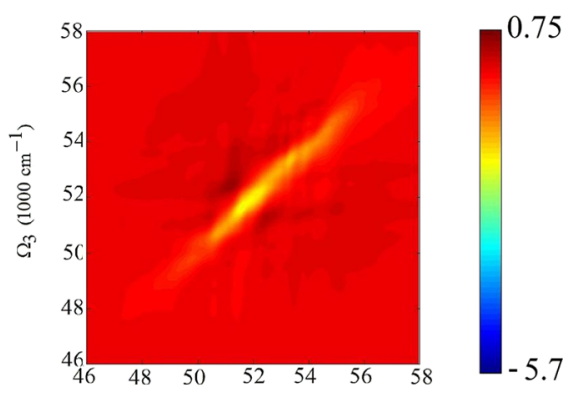

(f) $\quad-\Omega_{1}\left(1000 \mathrm{~cm}^{-1}\right)$

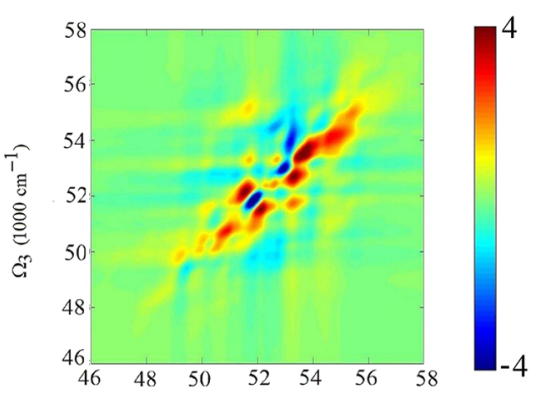

(i) $\quad-\Omega_{1}\left(1000 \mathrm{~cm}^{-1}\right)$

Figure 6. Contributions of the turns and the $\beta$-strands to the 2DFUV signal with the $x y x y-x y y x$ polarization configuration dissected by means of different methods. (a) The sum of parts $\mathrm{b}$ and $\mathrm{c}$. In part $\mathrm{b}$, a Frenkel exciton Hamiltonian of the $\beta$-strands is separated from the Hamiltonian of the whole aggregate model. (c) The same approach for the turns (the signal is 1 order of magnitude lower than the one in part b). (d) The sum of parts e and f. (e) A dissection by projecting the last light-matter interaction of the four wave mixing experiment in real space ${ }^{15}$ is applied to the $\beta$-strands. (f) The same approach for the turns. (g) The sum of parts h and i. (h) A sensitivity analysis as described in section 5 is applied to the diagonal elements of the Hamiltonian associated with the $\beta$-strands. (i) The same approach is applied to the turns.

$50000 \mathrm{~cm}^{-1}$. The signal for the turns is again shown to be dominant around the diagonal, consistent with the argument on the splitting of amide $\pi \rightarrow \pi^{*}$ transitions.
A one-dimensional section of the 2DFUV spectrum is shown in Figure 7, with the corresponding contributions of the turns and $\beta$-strands. This section is along the antidiagonal 

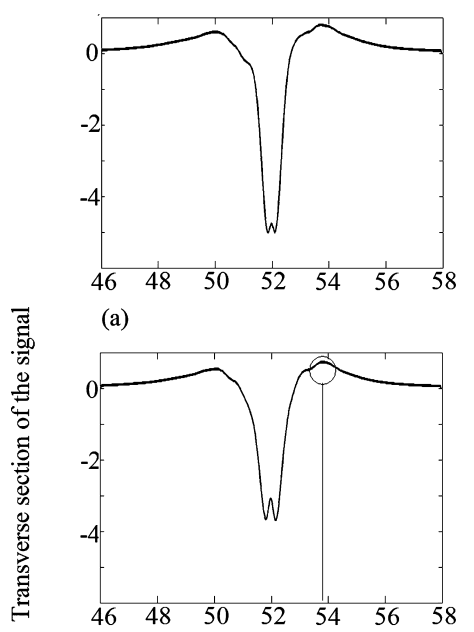

(b)

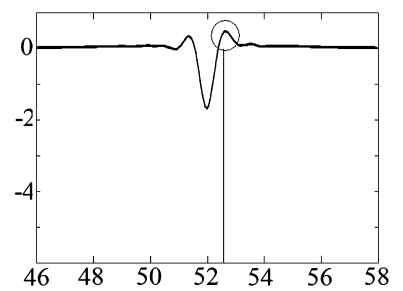

(c)

$$
-\Omega_{1}\left(1000 \mathrm{~cm}^{-1}\right)
$$

Figure 7. Section of the $x y x y-x y y x$ 2DFUV signal in the diagonal direction (from 46000 to $58000 \mathrm{~cm}^{-1}$ along $-\Omega_{1}$ and from 58000 to $46000 \mathrm{~cm}^{-1}$ along $\Omega_{3}$ ): (a) for the whole system (from Figure $6 \mathrm{~d}$ ); (b) for the $\beta$-strands (from Figure 6e); (c) for the turns (from Figure 6f). Cross-peaks for the $\beta$-strands and the turns are highlighted using red lines.

(from 46000 to $58000 \mathrm{~cm}^{-1}$ along $-\Omega_{1}$ and from 58000 to $46000 \mathrm{~cm}^{-1}$ along $\Omega_{3}$ ). The signal is much broader at the $\beta$-strands, as expected due to the larger delocalization in this region (see section 4). As shown in Figure 7a, there is a central negative peak at $52000 \mathrm{~cm}^{-1}$ and two positive small peaks at around 50000 and $54000 \mathrm{~cm}^{-1}$. The central peak consists of contributions from the turns and $\beta$-strands, and the other two peaks are comprised of contributions form the $\beta$-strands only. The magnitude of the ratio of the central peak and the peak at $50000 \mathrm{~cm}^{-1}$ is a measure of the extent of turns relative to strands in the system. When the ratio is calculated only for the contribution of the strands, Figure $7 \mathrm{~b}$, it is 6 . This corresponds to the value for which the contribution of the turns vanishes. For the ratio calculated for the entire spectrum, Figure $7 \mathrm{a}$, the contribution of this term is 8 . This may be used to quantify environmental factors that affect differently the strands and the turns of the proteins, and the conformational evolutions that may be experienced by the proteins during the fibril growth. $^{22}$

As indicated in section 3 , the angles between neighboring amide $\pi \rightarrow \pi^{*}$ transition dipoles are almost constant at the $\beta$-strands and it is $100^{\circ}$ for most neighboring transition dipole pairs. The magnitude of the $x y x y-x y y x$ signal is known to be maximum for $90^{\circ}$, and consequently, the signal is increased by the geometry at the strands. The angles between amide $\pi \rightarrow \pi^{*}$ transition dipoles at the turns fluctuate drastically during the molecular dynamics simulation between $20^{\circ}$ and about $140^{\circ}$. These fluctuations are expected to weaken the signal, as the angles are generally away from $90^{\circ}$, and they will also broaden the contribution of the turns to the signal.

\section{CONCLUSIONS}

We have illustrated the properties of excitons at the $\beta$-strands and turns of an amyloid fibril model, and discussed how these segments contribute to the 2DFUV signal. Exciton splitting is much larger for the strands, and they generate cross-peaks farther from the diagonals than the turns. One central peak and two cross-peaks are the main features expected for proteins. The ratio of the intensity of the central peak and the crosspeaks is useful for the characterization of the secondary structure. $\beta$-strands show strong cross-peaks, while the turns show a relatively intense central peak. The detection of transport using the photon echo signal is expected to inform on further details of the secondary structure.

\section{AUTHOR INFORMATION}

\section{Corresponding Author}

*E-mail: rodriguez.justo@gmail.com.

\section{Notes}

The authors declare no competing financial interest.

\section{ACKNOWLEDGMENTS}

We gratefully acknowledge the support of the National Institutes of Health (grant GM059230) and the National Science Foundation (grant CHE-1058791). We wish to thank Dr. Alfonso Lam and Dr. Jun Jiang for helpful discussions.

\section{REFERENCES}

(1) Brahms, S.; Brahms, J. J. Mol. Biol. 1980, 138, 149.

(2) Besley, N. A.; Hirst, J. D. J. Am. Chem. Soc. 1999, 121, 9636.

(3) Bulheller, B. M.; Miles, A. J.; Wallance, B. A.; Hirst, J. D. J. Phys. Chem. B 2008, 112, 1866.

(4) Ajdarzadeh Oskouei, A.; Bram, O.; Cannizzo, A.; van Mourik, F.; Tortschano, A.; Chergui, M. J. Mol. Liq. 2008, 141, 118.

(5) Ajdarzadeh Oskouei, A.; Bram, O.; Cannizzo, A.; van Mourik, F.; Tortschano, A.; Chergui, M. Chem. Phys. 2008, 350, 104.

(6) Tseng, C.; Matsika, S.; Weinacht, T. C. Opt. Express 2009, 17, 18788.

(7) Beutler, M.; Ghotbi, M.; Noack, F.; Brida, D.; Manzoni, C.; Cerullo, G. Opt. Lett. 2009, 34, 710.

(8) Corkum, P., De Silvestri, S., Nelson, K. A., Riedle, E., Schoenlein, R. W., Eds. Ultrafast Phenomena XVI; Springer: New York, 2009.

(9) Selig, U.; Schleussner, C.-F.; Foerster, M.; Langhojer, F.; Nuernberger, P.; Brixner, T. Opt. Lett. 2010, 35, 4178.

(10) Jiang, J.; Mukamel, S. Phys. Chem. Chem. Phys. 2011, 13, 2394.

(11) Zanni, M. T.; Ge, N.-H.; Kim, Y. S.; Hochstrasser, R. M. Proc. Natl. Acad. Sci. U.S.A. 2001, 98, 11265.

(12) Ginsberg, N. S.; Davis, J. A.; Ballottari, M.; Cheng, Y.-C.; Bassi, R; Fleming, G. R. Proc. Natl. Acad. Sci. U.S.A. 2011, 108, 3848.

(13) Voronine, D. V.; Abramavicius, D.; Mukamel, S. J. Chem. Phys. 2007, 126, 044508.

(14) Voronine, D. V.; Abramavicius, D.; Mukamel, S. J. Phys. Chem. 2010, 115, 4624.

(15) Abramavicius, D.; Palmieri, B.; Vorinine, D. V.; Sanda, F.; Mukamel, S. Chem. Rev. 2009, 109, 2350.

(16) Zhuang, W.; Abramavicious, D.; Mukamel, S. Proc. Natl. Acad. Sci. U.S.A. 2005, 102, 7443.

(17) Petkova, A. T.; Yau, W. M.; Tycko, R. Biochemistry 2006, 45, 498.

(18) Bulheller, B. M.; Rodger, A.; Hirst, J. D. Phys. Chem. Chem. Phys. 2007, 9, 2020.

(19) Platt, J. R. J. Chem. Phys. 1949, 17, 484. 
(20) Kurapkat, G.; Krupger, P.; Wolimer, A.; Fleischhauer, J.; Kramer, B.; Zobel, A.; Koslowski, A.; Botterweck, H.; Woody, R. W. Biopolymers 1997, 41, 267.

(21) Economou, E. N. Green's Functions in Quantum Physics; Springer: New York, 1994.

(22) Rojas, A.; Liwo, A.; Browne, D.; Scheraga, H. A. J. Mol. Biol. 2010, 404, 537. 